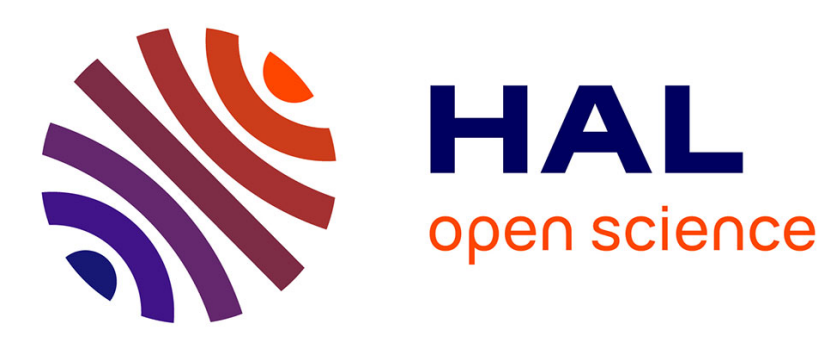

\title{
SOINS PRECOCES ET GUIDANCE PARENTALE : LE POINT DE VUE DES FAMILLES
}

\author{
Annick Camille Dumaret
}

\section{To cite this version:}

Annick Camille Dumaret. SOINS PRECOCES ET GUIDANCE PARENTALE : LE POINT DE VUE DES FAMILLES. Dialogue, 2003, 162, pp.9-24. halshs-00353239

\section{HAL Id: halshs-00353239 \\ https://shs.hal.science/halshs-00353239}

Submitted on 15 Jan 2009

HAL is a multi-disciplinary open access archive for the deposit and dissemination of scientific research documents, whether they are published or not. The documents may come from teaching and research institutions in France or abroad, or from public or private research centers.
L'archive ouverte pluridisciplinaire HAL, est destinée au dépôt et à la diffusion de documents scientifiques de niveau recherche, publiés ou non, émanant des établissements d'enseignement et de recherche français ou étrangers, des laboratoires publics ou privés. 
Paru dans Dialogue, $n^{\circ} 162,4^{\text {ème }}$ trimestre 2003, $p$ 9-24

\title{
SOINS PRECOCES ET GUIDANCE PARENTALE : LE POINT DE VUE DES FAMILLES
}

\author{
Annick-Camille DUMARET
}

Avec le développement de la psychiatrie périnatale, les prises en charge précoces des familles se sont diversifiées. Au fil du temps, des équipes se sont efforcées de faciliter l'accès aux soins à des familles peu enclines à y adhérer d'elles-mêmes. Des institutions se sont organisées pour aider des familles à haut risque psychique, dans le but de lutter contre les processus inter-générationnels qui induisent maltraitance et placements. D'autres ont créé des lieux de parole et d'échange initiant des stratégies de rencontre permettant aux familles de rompre peu à peu avec l'exclusion.

Les familles adressées dans ces structures sont surtout des familles connues des services sociaux, pédiatriques, juridiques et aussi des services de psychiatrie d'adultes. On peut s'interroger à la fois sur la manière dont les parents ont perçu les propositions qui leur ont été faites et également sur le regard porté sur eux par les professionnels des équipes soignantes et sociales. Ce regard détermine en partie leur adhésion au type de soins proposés. Comme l'ont montré des recherches américaines, l'implication des familles va conditionner fortement la réussite des programmes d'interventions précoces et d'accompagnement (Dumaret 2003). Pour progresser dans ce domaine et améliorer les prises en charge, des évaluations doivent être menées, même si chaque institution ou chaque population traitée est unique, même si l'on ne dispose pas de "groupe contrôle». On peut, si l'on s'en donne les moyens, étudier le devenir des familles à partir d'outils standardisés (questionnaires socio-démographiques, tests, échelles) complétés par des approches qualitatives. De quels autres critères d'évaluation dispose-t-on? Il est intéressant de partir du point de vue des usagers pour apprendre, même si c'est dans l'après-coup, ce dont ils ont bénéficié et repérer la manière dont ils se sont réapproprié les soins reçus. Ce qui est le plus thérapeutique n'est pas forcément ce que l'on croit, le point de vue des professionnels sur ce qui peut être défini comme une amélioration de la situation n'est sans doute pas exprimé de la même façon par les usagers.

Plusieurs institutions axées sur les dysfonctionnements de la relation parents-enfants et la lutte contre la maltraitance ont fait l'objet d'un travail évaluatif dans le cadre d'un programme de recherche mené au CERMES. L'option retenue ici est de présenter deux de ces structures de soins dont l'objectif initial est assez identique (traiter la parentalité défaillante, oeuvrer pour la (re)construction des liens familiaux et le développement des potentialités de l'enfant) mais dont les modalités d'intervention et de soins sont différentes. Il s'agit de l'Unité de Soins à Domicile de Jeunes Enfants du XIII ${ }^{\text {ième }}$ arrondissement de Paris et d'une activité particulière du CAMSP de Roubaix. L'une va se centrer sur le bébé ou le petit enfant en intégrant la famille et son environnement, l'autre se centre en premier lieu sur les parents et leurs potentiel de compétences. Alors qu'il n'y a pas d'assistante sociale à l'Unité de Soins où les soins psychiatriques sont différenciés du soin social, celle-ci est présente au groupe du mardi dont 1'une des préoccupations principales est de traiter l'exclusion avant de faire de l'action médicosociale. Par ailleurs, les références théoriques des équipes sont également différentes. Ceci rejoint les résultats d'études sur les pratiques des professionnels de la petite enfance dans des lieux centrés sur les relations mère-enfant; ces pratiques s'appuient sur des théories relevant de deux tendances : l'une, issue de la psychanalyse, insiste sur un travail individuel, 1'autre, alimentée par la psychosociologie, se fonde sur le principe de la socialisation (Donati 1999). Cet article est centré uniquement sur les familles très carencées à risque psychosocial 
et psychiatrique que j'ai interviewées (note 1). Par delà les protocoles de soins, j'essaierai de faire ressortir à la fois les convergences et les différences relevées dans le discours des familles suivies.

\section{Des familles à problèmes multiples}

Dans ces milieux défavorisés sur le plan socio-économique et sur le plan de la santé, les parents vivent des situations de détresse multifactorielle (David et col. 1984). Ces familles sont fragilisées par l'engrenage intergénérationnel de la misère, l'accumulation des problèmes économiques et les risques psychosociaux. Nombre de parents ont déjà expérimenté dans leur enfance des placements et des séparations, ils ont vécu les hospitalisations de leurs propres parents et/ou des décès dans leur fratrie. Ces traumatismes ont rarement pu être élaborés mentalement et les sentiments associés à ces expériences n'ont pu se manifester faute de moyens d'expression. En outre, les dépressions parentales sont couvent masquées.

L'accès à la parentalité est une période de transition à risque qui va réactualiser des difficultés au niveau du couple, réactiver des angoisses archaïques chez les futures mères, la plupart isolées et issues de familles désorganisées, avec des aînés vivant souvent hors du milieu familial à cause de risques de maltraitance (Gauthier 1998). Le manque de repères, l'absence de références positives renvoient ces parents à des sentiments de culpabilité, d'incompétence et de mésestime de soi. Ils ont parfois renoncé à demander de l'aide face à la parcellarisation des prises en charge proposées par les différents services, eux-mêmes démunis et découragés. Des parents font preuve d'incohérence dans les soins primaires et l'organisation de leurs rythmes, d'autres ne peuvent les assumer. Aussi les enfants vivent-ils de graves discontinuités dans les soins ; la majorité présentent des troubles du développement et de la communication, un déficit d'échanges langagiers, sensoriels, moteurs, émotionnels... Leurs souffrances sont intriquées à celles de leurs parents et ces familles vivant dans une insécurité constante sont peu à l'écoute de leurs enfants et peu attentives à leurs besoins. Les interactions mère-bébé sont décrites par les professionnels comme pauvres ou évitantes, les investissements souvent violents et excitants. L'enfant est parfois vécu comme persécuteur, ou encore comme un partenaire sexuel en raison des manques affectifs des parents.

Les familles rencontrées présentaient d'importants facteurs de risque : violence conjugale et parentale, conduites alcoolo-dépendantes pour la moitié des mères dans les deux lieux et la plupart des pères dans le Nord, climat incestueux et abus sexuels dans moins de la moitié des situations à Paris mais en proportion un peu plus élevée dans le Nord. Malgré de nombreux facteurs de risque communs, les configurations cliniques sont diverses et complexes: les pathologies dépressive et borderline dominent et à ce tableau s'ajoute la déficience intellectuelle, l'absence de maîtrise du langage et de l'abstraction. Certains parents, ayant été peu scolarisés ne sont pas immédiatement prêtes à un travail psychologique fondé sur la parole. C'est d'un soutien social dont ils ont besoin en premier lieu ; il est nécessaire d'établir une relation basée sur la confiance avec ces mères et ces pères pour lesquels la question de la rupture des liens a souvent été centrale dans leur vie.

\section{Des modalités d'intervention très différentes}

Deux institutions, 1'Unité de Soins Spécialisés à Domicile de jeunes enfants dirigée entre 1983 et 2001 par le Docteur Françoise Jardin, psychiatre et psychanalyste, et le CAMSP de Roubaix dirigé par le Docteur Maurice Titran, pédiatre, depuis 1981, ont été des pionnières dans leur conception du travail avec les familles : accès aux soins précoces, interventions à domicile et accompagnement, permettant une véritable alliance thérapeutique. Dans ces contextes qui prennent en compte la globalité de l'entourage du jeune enfant, les liens avec les 
familles ont été travaillés pour que se (re)construise une histoire, pour soutenir le processus de parentalité (aider les adultes à devenir les parents de cet enfant-là) et favoriser le développement psychique et la santé physique de l'enfant. Les modalités d'intervention diffèrent et sont fonction des références théoriques : d'un côté, les soins sont fournis par des professionnels et de l'autre, des parents et des bénévoles collaborent avec des professionnels à une action commune. Aussi bien à 1'Unité de Soins qu'au CAMSP, les équipes sont multidisciplinaires et travaillent dans la continuité depuis de nombreuses années. Un travail est mené en partenariat avec les équipes sociales et médicales (pouponnières, placements familiaux, PMI, SSE, crèches, écoles), celles de la justice et des services d'hygiène mentale adulte.

\section{L'Unité de soins spécialisés pour jeunes enfants : une approche clinique}

C'est le seul SSEAD (service d'éducation spécialisée et de soins à domicile) psychiatrique du département de Paris pour parents et jeunes enfants. Son action préventive et thérapeutique concerne à la fois l'enfant, sa mère, le lien mère-père-enfant et son environnement. Le travail se fait souvent, au début du moins, aux frontières du social et du soin psychique mais a pour objectif 1 'accès à la vie psychique du jeune enfant et le soutien à la parentalité. Les modes de travail ont été largement décrits par ailleurs (David et Jardin 1993, Lamour et Barraco 1998, Jardin et Detry 2003). Les références théoriques sont la psychanalyse et la théorie de l'attachement avec la clinique des interactions. Le travail «direct» auprès des familles s'effectue à partir de consultations familiales. Parallèlement aux traitements habituels de type psychothérapique et aux rééducations (orthophonie, etc.), des outils spécifiques de soin ont été développés : l'observation du bébé, le travail à domicile, les accompagnements thérapeutiques, les groupes à visée thérapeutique, groupes d'enfants, groupes de parents... Le traitement des situations de séparation parents-enfant est longuement préparé et accueilli par les parents comme un soin et non comme une sanction. Ainsi, peu à peu les modalités de traitement des parents, du bébé et du lien s'organisent au cours des rencontres et le traitement est modulable en fonction de l'évolution et de la demande. En ce qui concerne plus particulièrement les familles carencées (la moitié d'entre elles exactement), parallèlement au travail d'accompagnement vers l'extérieur (éducateurs et infirmiers) et au travail thérapeutique à domicile (celui des parents ou celui de l'assistante maternelle), a lieu la participation à des groupes de parents et parents-enfants. «Le travail «indirect» en commun avec les équipes sociales et médicales permet de repérer les clivages dans le fonctionnement maternel, dans la relation mère-enfant et entre les professionnels et de dégager les professionnels des impacts émotionnels engendrés par les situations familiales. «Le travail est alors une réflexion sur la place de chacun et une aide pour chacun à définir et à retrouver son identité professionnelle, ses compétences, ses limites» (Blondel et Jardin 1997). L'équipe est mobile et se déplace si nécessaire sur les différents lieux de vie des enfants (crèches, haltegarderie, familles d'accueil). Le dispositif d'ensemble est coordonné par un consultant.

\section{Le groupe du mardi : une approche psychosociale}

Le travail du CAMSP consiste en premier lieu à aider ces familles à (re)trouver confiance dans les professionnels, ce qui a été traduit par "l'évaluation des compétences et l'estime réciproque» (Titran 1994). Ce n'est qu'après qu'un projet pour l'enfant sera impulsé en accord avec les parents et les autres partenaires sociaux. C'est dans ce cadre qu'est né, il y a plus de quinze ans, le " groupe du mardi », auquel participent les parents, des bénévoles et des professionnels. Il s'adresse en exclusivité à «des familles qui ne se sentent incluses ni dans l'action médicale, ni dans l'action sociale, ni dans l'action pédagogique, ni dans l'action éducative, ni dans l'action culturelle " (Dumont 2000). C'est un " groupe informel qui se fait sur un mode convivial; il est un lieu d'échange, d'expression, de connaissance et de 
reconnaissance de l'autre " (Titran 1993). Il fonctionne un après midi par semaine, tout au long de l'année, même pendant les vacances scolaires. Sa finalité consiste à restaurer une identité sociale, une image de soi «suffisamment bonne» et à valoriser les compétences parentales par de petits actes ordinaires. Les professionnels (côté parents et enfants) et les bénévoles qui accompagnent ces familles sont toujours les mêmes. Dans son fonctionnement, il est très tolérant vis à vis des comportements des parents, sauf en cas de violence. C'est aussi un lieu de retrouvailles pour des parents dont les enfants sont placés par décision judiciaire. Des activités de la vie quotidienne s'organisent avec un partage de temps et de savoir. Au sein $\mathrm{du}$ groupe, l'écoute et l'observation des interactions mère-enfant permettent aux professionnels d'identifier ce dont les familles ont besoin. Par son enveloppe structurante et bienveillante, le groupe offre aux familles la possibilité de fonctionner avec des repères. Les activités manuelles libèrent peu à peu la parole qui va circuler dans le groupe où chacun peut faire valoir ses compétences; un repas en commun est organisé mensuellement avec ces familles qui peuvent bénéficier par ailleurs d'une semaine de vacances en maison familiale. Une réunion de synthèse a lieu à la fin de chaque séance de groupe, réunissant professionnels et bénévoles pour faire le point sur l'évolution de l'enfant; elle représente un espace de régulation des interactions et des tensions qui ont pu être observées lors de la séance.

\section{La rencontre avec ces familles}

L'échantillon de familles rencontrées ne reflète pas l'ensemble des populations soignées mais concerne celles qui avaient de très jeunes enfants et qui ont été suivies pendant plus d'un an. Ces prises en charge de l'enfant et de sa famille ont duré deux fois plus longtemps à 1'Unité de Soins (soit en moyenne six ans) qu'au groupe du mardi du CAMSP de Roubaix. Le programme de recherche comportait une évaluation psychosociale des situations familiales actuelles et une évaluation plus clinique des enfants au moins cinq ans après la fin des soins (note 2). L'abord de ces familles avait été étudié soigneusement avec les équipes : elles ont été contactées directement par un courrier du directeur du lieu de soins qui sollicitait leur participation à la recherche. Peu nombreuses sont les familles venues d'elles-mêmes dans 1'institution; pour les autres, c'est un professionnel bien connu des parents (une infirmière psychiatrique retraitée pour l'Unité de Soins et l'assistante sociale ou le pédiatre pour le CAMSP) qui s'est rendu au domicile et leur a présenté la personne devant les interviewer ; il leur était précisé qu'elle était totalement indépendante de l'institution et que la confidentialité des entretiens était garantie. Il y a eu très peu de refus de la part de ces familles; les parents ont accepté de participer à la recherche, vraisemblablement par désir de rendre quelque chose à ceux qui les avaient aidés ou de montrer les aspects positifs de leur évolution. Très peu de pères ont été rencontrés, certains avaient une activité professionnelle, des couples s'étaient séparés ; ces pères sont généralement plus fuyants et certains ont été écartés de leur famille. Ce sont les mères venues au groupe du mardi qui ont accepté le plus facilement les rencontres, lesquelles ont généralement eu lieu à leur domicile. Pour ces familles, le fait d'être venues autrefois dans un lieu où il y avait non seulement des soins mais aussi des groupes de parents, le fait aussi que le kinésithérapeute se rendait au domicile ainsi que des bénévoles, peut avoir facilité l'accueil du chercheur à leur domicile.

\section{Les objectifs des soins et de l'accompagnement du point de vue des parents}

Les souvenirs des parents sont assez vagues concernant les professionnels qui ont orienté les familles, mais souvent elles les nomment, elles parlent du médecin de la maternité, de la "crèche", de la "PMI", de la "rue M." où se trouve le service social du secteur. On trouve 
dans le discours des mères des confusions dans les événements qui ont eu lieu parfois plusieurs mois avant l'admission, ce que l'on peut relier à des périodes de vie très difficiles : climat de violence, précarité et errance, placement et hospitalisation de l'enfant. Dans plusieurs situations, les prises en charge se sont déroulées en continuité du travail qui avait commencé avec des aînés de la fratrie : "J'étais venue pour Kévin ils ont pris en même temps dans le lot ma fille qui commençait à peine à parler"; "On l'avait débranché du monitoring, c'était une enfant souriante qui avait une joie de vivre mais j'avais fait un blocage sur elle... J'étais venue pour les autres mais elle, elle était bien ". On peut penser que la mise en place des relations parents-enfant et de la parentalité, plus rapide, a été le fruit d'années de prises en charge antérieures. Par ailleurs, dans ces deux situations, les mères ont perçu la finalité préventive de cet accompagnement proposé par l'institution.

\section{Le besoin de montrer son attention à l'enfant}

La plupart des mères ne disent pas être venues pour leurs propres difficultés, elles n'ont souvent jamais pu parler, mettre des mots sur leurs souffrances : "J'avais peur d'aller voir un psychologue, de reconnaître que j'avais un problème "; " On tient tout à l'intérieur, on le dit à personne et après on fait une petite déprime. C'est ce que j'ai fait après la naissance de $P$. " Elles mettent en avant les problèmes de leur fille ou de leur garçon, avec des descriptions plutôt sommaires mais qui nous font cependant entrevoir les difficultés de l'enfant : "Il était sauvage "; "Elle était timide, elle allait pas vers les gens"; "Elle avait un problème de hanche "; "C'était pour qu'il parle ». D'autres peuvent apporter quelques détails qui montrent ainsi leur inquiétude parentale face à ce bébé souvent malade : " $C$. était toujours malade En fait, il prenait trop de médicaments et le docteur m'a proposé de venir au groupe "; ou souffrant de troubles somatiques : "Ma fille se cachait et se collait à moi "; "Elle était adorable mais de la naissance à huit mois j'ai jamais pu dormir "; "Il avait des tas de problèmes, il était toujours malade; il a fait une gastro à cinq mois et après il était soigné dans les hopitaux. Il ne voulait jamais manger quand je lui donnais le biberon, on aurait dit qu'il avait de la misère à avaler ». Quelques mères ayant des capacités d'expression verbale mais qui présentaient d'importants troubles psychiques, ont pu décrire avec précision leurs difficultés relationnelles de l'époque. Ainsi la mère de T. se souvient de cet enfant non désiré, conçu dans la violence : "Quand il est né, même que je le prenais au sein, il se mettait à hurler, pareil pour le bain, ça me tapait sur le système. J'ai très mal vécu ça, j'en ai parlé au docteur qui m'a donné rendez-vous [...]. Et après je suis vemue au groupe pour me libérer un peu, pour souffler, le médecin voulait le voir régulièrement, $T$. faisait de la psychomotricité et moi, je pouvais faire des activités parce que mon ex, il voulait pas que je sorte $)$.

Nombreuses sont celles qui ressentent le besoin de dire qu'elles ont été à l'initiative de cette aide envers leur petit enfant mais aussi qu'elles n'ont pas tout accepté : "Je suis passée dans le quartier et j'ai vu ce centre qui aidait les enfants, je suis entrée par hasard"; "Elle communiquait pas, donc il fallait que je l'aide un peu... les crèches, j'ai essayé, elle voulait pas y aller, elle pleurait comme si je l'abandonnais "; "Ma tutrice m'a dit, va voir. J'ai vu que ça m'intéressait et j'y suis retournée avec les enfants"; "Je venais pour lui, ils $m$ 'ont bien accueillie, mais...j'ai refusé qu'il aille au groupe des bébés ». Elles ont adhéré aux activités proposées auxquelles elles ont participé : "Elle nous demandait si ça nous intéressait de faire ceci ou cela ».

De façon générale, hormis les rééducations en orthophonie et en psychomotricité, les observations thérapeutiques en crèche et l'organisation de séjours vacances en familles d'accueil par l'Unité de Soins n'ont pas été mentionnés. On peut relier ces oublis à des souvenirs pénibles de séparation ou au désir de masquer leurs difficultés personnelles à s'occuper de leur enfant. On peut aussi penser que ces moments de prises en charge restent 
très extérieurs aux parents, ainsi que l'important travail de partenariat avec les autres professionnels (ASE, écoles...) qui n'a pu être perçu. Néanmoins, toutes ces mères se souviennent nommément de ceux qui sont venus à leur domicile et/ou de ceux qui les ont accompagnées à l'extérieur (psychiatres, infirmiers, éducateurs). Peu abordent la question de leur suivi individuel sauf quand la relation a duré assez longtemps comme cette mère dont les trois filles ont été suivies: "Mes soucis, je les garde ou j'explose, mais ça va sortir par dépression et je me retrouve sous traitement... Mon meilleur souvenir ?Je parlais de la dépression, c'est avec le docteur que je me parlais... Déjà à la maternité, elle me reprenait quand je disais IL, elle me reprenait à chaque fois, non ELLE! [cette mère avait désiré un garçon] ". Il en est de même de la description de l'ensemble des soins reçus; une mère décrit avec justesse la stratégie de soins adoptés pour elle et pour ses enfants : "Quand on est veuve, qu'on se retrouve seule, sans parents avec trois enfants, c'était, c'est difficile. On a toujours peur, on se dit: "est-ce que vraiment on va être mère hein, est-ce qu'on est capable de... Ils m'ont envoyé quelqu'un à domicile pour me montrer comment il fallait faire calmement... Et puis après je suis allée dans le groupe, avec mes garçons, et j'ai rencontré des parents et tout... Pour ma fille, dès sa naissance, je voulais la protéger comme son frère, comme elle connaissait pas son père, je voulais que si il $m$ 'arrive une tuile, qu'on $m$ 'aide à supporter... Donc on a trouvé des familles pour eux, ils partaient en vacances et moi, j'étais un peu plus libre (...). Je voyais Mme X. une fois par semaine toute seule pour tout mettre en ordre, tout ce que je pensais. Et j'accompagnais mes enfants en groupe et en même temps je discutais avec les parents, à l'accueil, le temps que les enfants ils travaillaient en groupe avec les moniteurs. "

Deux mères rencontrées s'autorisent à critiquer les soignants de 1'Unité de Soins par rapport aux horaires des thérapies (pendant les heures de classe), ou sur la grande liberté laissée aux enfants lors de jeux communs : "Ils avaient un peu trop de liberté, par exemple, elle mangeait un gâteau, elle [l'éducatrice] voyait un enfant taper dans le gâteau, elle trouvait ça tout à fait normal, non c'est pas bien. " Elles veulent montrer ainsi leur autorité parentale, et la difficulté des soignants à contenir les enfants. L'émergence d'une certaine capacité de critique semble plus notable dans ce lieu de soins que dans le groupe du mardi, sauf pour une mère qui demande pourquoi les mères ne participent pas à la réunion de synthèse entre professionnels et bénévoles.

\section{L'attachement aux activités de groupe}

Parmi les modalités de soins communes aux deux institutions, les activités de groupe sont très présentes dans l'esprit des parents, avec l'évocation du climat convivial des rencontres, le souvenir d'autres parents avec leurs enfants : "On allait dans la grande salle, on discutait, on regardait les gosses jouer avec d'autres gosses "; "On faisait connaissance, on fêtait les anniversaires... on parlait avec les autres mamans, j'ai des photos des mamans ». Non seulement on s'occupait de leur enfant, mais aussi d'eux. Dans ce lieu enveloppant et contenant, les parents retrouvent des émotions, ils se sentent exister, ils régressent parfois en se mettant à jouer: "On rigolait bien, on pouvait s'éclater, jouer avec eux », autant d'attitudes impossibles au domicile avec le conjoint. Les mères dont les enfants étaient déjà en placement se rappellent de ces accompagnements entre le domicile et les lieux fréquentés par l'enfant, comme la pouponnière, la halte-garderie, la crèche : " $N$. allait chercher ma fille à la maternelle et elle l'accompagnait à l'Unité » ou d'avoir vu leur enfant au groupe du mardi : "Je savais que ça se passait bien chez la nourrice, elle venait ici avec elle, c'est comme ça que je la voyais ".

Les familles du Nord parlent avec chaleur de ces rencontres qui commençaient en début d'après midi avec le café et leur permettaient peu à peu de sortir de l'isolement : "Dans le ménage de mes parents, je parlais à personne. Là, au moins je n'étais pas seule chez moi à 
broyer du noir "; "Ils travaillaient avec les enfants et nous on discutait... Ca m'évitait de penser à des trucs que j'ai vécus quand j'étais chez mes parents »; "C'était régulier, c 'était bien toutes ces activités, ça nous changeait de l'ordinaire, sinon on sortait pas de la maison"; "Ca permettait aux gosses de voir autre chose que leur quartier... les enfants étaient en contact avec d'autres, c'était l'occasion de les voir évoluer ensemble. "Les activités manuelles contribuent par l'intermédiaire des interactions au partage des compétences individuelles et à la construction sociale de l'identité. Elles renforcent aussi la solidarité, notion très marquée dans leur mémoire : "On faisait des nounours, des trucs de Pâques... et tout ça, on le vendait pour pouvoir permettre aux autres de partir en vacances une semaine. " Cette spécificité du groupe, à travers une activité productrice de gestes de la vie quotidienne - les activités manuelles - est un support aux échanges verbaux, un préalable indispensable pour ouvrir vers la réflexivité. Le groupe va permettre de découvrir une certaine capacité à rêver et à penser que l'on peut soi-même évoluer : "Je me disais, si elle s'en est sortie, pourquoi pas moi? ».

\section{Perception d'un regard bienveillant des soignants et sentiment de confiance à l'égard du groupe}

Le regard des équipes soignantes sur les familles est d'autant plus important que la société leur renvoie une image dévalorisante d'elles-mêmes : "Aussitôt que je sortais d'ici, on me regardait comme si j'étais une clocharde. Ici, on m'écoute, je suis considérée " dit une mère. Le regard porté par les soignants a été perçu comme chaleureux et empathique : des parents considérés comme des parent ayant leurs compétences, et non des patients ou des parents maltraitants. Les enfants, eux aussi ont perçu le regard porté par les adultes sur leurs parents, ils ont découvert que leur mère pouvait être appréciée pour ses talents de brodeuse, etc...

Des mères ont découvert à l'Unité de Soins qu'elles pouvaient être accueillies en tant que personnes par un regard bienveillant qui ne les juge pas : "Je me souviens avoir dit lors d'un premier rendez-vous au docteur 'j'ai eu chand' parce que effectivement j'étais déprimée. Ca m'a rassérénée, ça m'avait remise sur pied un peu, j'avais chaud, de la chaleur humaine. " ; "Elle m'a beaucoup aidée à remonter le moral. Quand j'avais un problème je pouvais l'appeler à n'importe quelle heure... tout le monde a été gentil avec moi. Et c'est grâce à elle que je suis devenue beaucoup plus mère, plus mîre avec eux [ses enfants]. Et après, c'est grâce à eux que j'avais confiance en ce groupe, elle m'a beaucoup rassurée. Et moi j'avais toujours peur, je parlais trop de mon enfance, j'avais toujours peur qu'on me prenne mes enfants... moi j'ai été tellement à la DDASS. J'avais peur qu'on me prenne mes enfants parce que j'avais pas de travail».

Chaque parent, le plus souvent la mère et parfois le père, vient à son propre rythme au groupe du mardi. Se faire accepter par les autres est nécessaire, et le groupe va jouer ce rôle d'ouverture, tout en canalisant les comportements (note 3). Le processus qui se met en place et qui nécessite l'instauration d'une véritable solidarité et de la confiance est bien expliqué : "Y en a qui parlaient, y en a qui parlaient pas, ils étaient gênés. On se demandait au début où on était... on ne connaissait personne et puis on se met un peu à la fois dans le bain, on commence à parler, on arrive à se conseiller l'un l'autre "; "Quand une nouvelle arrivait, on faisait les chiens de faïence, on se mettait sur nos gardes... et après on voyait comment elle parlait, comment elle était, on voyait si on pouvait l'aider, l'orienter ou si c'était un gros problème qu'on pouvait pas résoudre, alors on en discutait à l'écart... et on allait voir les autres [l'institutrice spécialisée, le kinésithérapeute...]». Alors que toutes les personnes rencontrées ont une grande reconnaissance envers 1'assistante sociale, cette mère reste méfiante encore aujourd'hui envers celle qui est en lien avec les services sociaux (non 
seulement ses aînés avaient été placés, mais également deux autre filles adolescentes à leur demande). Avec le temps, les angoisses vont s'exprimer, le désir d'entraide aussi, des liens se créent, des secrets enfouis se dévoilent: "On parlait même des choses très personnelles, violentes, vécues, vous voyez ce que je veux dire ». C'est avec reconnaissance que plusieurs mères, qui avaient raconté $\mathrm{x}$ fois leur histoire aux travailleurs sociaux, à la police, au juge..., mentionnent la discrétion de leurs rencontres : "Quand on avait un problème, on pouvait en parler avec le Dr. et les autres parents. On pouvait se confier et l'autre mettait pas ce qu'on disait ailleurs "; "On parlait avec les mamies de nos problèmes à la maison mais avec le Dr., on parlait d'autres choses. Entre nous, on se confiait beaucoup, on savait très bien que les autres mamans n'allaient pas en parler à des professionnels \%. Le maintien de cette confidentialité a été constaté au moment de l'enquête lors des entretiens avec des bénévoles qui n'ont dévoilé ni l'identité ni 1'histoire des personnes venues au groupe (note 4).

Des parents vont échanger leurs compétences, il y a celles qui savent coudre et broder, celles qui savent donner le biberon. Ainsi le partage de compétences ordinaires, peu impliquantes personnellement conduit à un partage de compétences parentales; remarques et conseils des uns et des autres sont alors acceptés. Par ailleurs, la transmission des savoir-faire se fait aussi entre familles et soignants, le médecin va affiner son expertise pédiatrique en écoutant la mère parler de ses conditions de vie et difficultés quotidiennes. Pour avoir vécu des situations similaires, des parents vont se retrouver dans la parole de l'autre; par exemple, des mères pourront parler de leurs souffrances d'enfant, révéler un secret caché jusque-là, elles choisissent leur interlocuteur, celui avec lequel elles ont une affinité : "Les premiers jours, c'est pas évident de parler... et après dans le domaine [sous-entendu, violence conjugale, difficultés de l'enfant...], automatiquement, on trouve quelqu'un à qui parler...Au début, je parlais pas du tout, j'ai été élevée comme ça, j'étais renfermée, je faisais de la dépression sans arrêt, j'avais plus confiance en personne. "Ce groupe, qui ne les juge pas et ne les met pas en échec, valorise leurs compétences et les renarcissise. La solidarité va se poursuivre hors du groupe pour certaines, même encore actuellement: "quand je me suis retrouvée aux urgences, elle est venue $m$ 'épauler ». Une mère est devenue la marraine d'une fillette d'une autre mère...

\section{Les représentations de l'aide reçue}

La majorité des personnes, qui ont suivi des thérapies individuelles avec ou sans leur enfant ou une thérapie familiale, ont noté le bienfait de ces soins pour l'enfant: "C'est surtout lui que ça a aidé ", ou pour elles: "Ca ne me disait rien. Bon c'est vrai une fois qu'on en parle, on est pas très bien et puis... toujours parler de ses problèmes, je pense pas que ça... Moi, ce qui me sort de mes dépressions, c'est de parler de mes enfants.... Je sais pas, je me bats pour eux. Je pense que c'est eux qui peuvent m'apporter. "

\section{L'aide aux parents}

Aujourd'hui, plus de la moitié de ces mères peuvent aborder leur histoire, parler de leur passé difficile, de leurs parents et de leur enfance, de ce petit frère ou de ce fils décédé subitement. Cette capacité à parler peut être mis en relation avec les soins précoces et les accompagnements. De même pour celles ayant participé aux rencontres avec d'autres parents : "On sentait qu'on était pas tout seuls à avoir des petites misères ». Certains ont noté qu'écouter les difficultés d'autrui demande un effort mais permet de réfléchir, on se décentre de ses propres problèmes, on cherche à aider: "On est écouté, aidé. C'était dur d'écouter, des fois, c'est très dur et on aimerait trouver une solution pour dire comment faire pour aider, on était solidaires. " Plusieurs utilisent le mot «libéré» pour signifier qu'elles ont pu exprimer des émotions, des souffrances ont été verbalisées : "On a pu parler, se libérer 
des problèmes qu'on avait. " Certaines mères ont le sentiment d'avoir eu une part active dans la protection de leur enfant, ce qui renforce l'image maternelle qu'elles se sont construites d'elles-mêmes : "J'étais contente de venir parce que j'étais libérée de mon mari et c'était pour sauver mes enfants, il était violent. Maintenant les enfants sont heureux. "

Le passage par le groupe a permis à certaines mères d'entreprendre une cure de sevrage, sachant qu'elles bénéficiaient du regard chaleureux et encourageant des autres parents, et à d'autres de faire la démarche d'aller consulter un psychologue ou un psychiatre. Avant le groupe, une mère avait tenté cette démarche sans succès, bloquée dans cette relation de face à face : "Elle [la psychologue. ] a dit c'est même pas la peine. Y'a rien qui sortait, j'arrivais pas à... En ce temps là je parlais pas du tout ». Par la suite, elle pense qu' "avec les autres parents, on a appris à se conseiller... je voulais $m$ 'en sortir, j'avais envie de comprendre. " Une autre mère, dont le fils venait de faire une tentative de suicide, a perçu sa demande : " $I l$ a besoin qu'on l'entende. Et peut-être que moi-même en tant que maman, il me lance des appels... Donc, même moi aussi j'ai besoin d'un travail comme ça avec mon fils, on est à trois avec le pédopsy... Parce que discuter avec des amis ça sert à rien parce que des amis ils n'ont pas l'objectivité d'une autre personne, d'une personne totalement étrangère. "

La plupart des parents, surtout les mères, évoquent avec plaisir ces rencontres qui leur ont apporté protection et les ont intégrées dans une vie sociale : "C'était peut-être la sécurité, de ne plus être seule, un peu du bien être... Ca m'a permis de rester en surface, carrément, sentir qu'on existe. Avant le groupe, j'avais plus envie de vivre... On reprend contact avec les gens. " Pour certains parents, la régularité de la venue et de la participation au groupe leur a offert des repères et une certaine normalisation : " Ca $m$ 'a appris de rester stable ... Quand mes enfants étaient à l'école, je sortais, j'étais un pigeon voyageur, je restais pas en place, j'allais me promener. " La restauration narcissique a contribué à relancer le développement des compétences, notamment des compétences parentales: "Je suis allée à la mer dans une maison de famille avec mes enfants. J'étais pas avec mes parents, donc je pouvais me débrouiller toute seule avec eux", de redécouvrir des compétences de la vie quotidienne, celles transmises autrefois au sein de la famille: "Je répare les habits de mes enfants et ma fille, je vais lui apprendre à coudre. Maintenant, je fais de la cuisine, avant je faisais toujours la même chose" ". Avec la revalorisation progressive des parents dans leurs compétences parentales, les réponses aux besoins des enfants sont devenues plus adéquates. Non seulement les parents devenus autonomes, mais aussi ceux dont la situation s'est améliorée et stabilisée (note 4) ont acquis une certaine confiance dans l'avenir et la possibilité de faire des projets ; ils se sont ouverts au monde extérieur. Un père dira : "C'était un vrai soutien moral... Ces voyages organisés, c'est ça qui fait qu'après j'ai pris mon envol, du fait que j'ai passé mon permis de conduire...."

La présence au groupe de bénévoles d'un certain âge déprofessionnalise l'aide apportée, offre aux familles une figure maternelle gratifiante et fait redécouvrir les différences entre générations : "Pour rien au monde, j'aurais loupé, pour passer une bonne après-midi avec les deux mamies [infirmière et éducatrice retraitées]. On pouvait discuter de nos problèmes... C'était une période heureuse quand je voyais mamie. Elle était tout pour moi, je la considérais comme ma grand-mère, comme ma maman, parce que y a des trucs de ma maman que je peux pas discuter " dit cette femme qui vit des relations fusionnelles avec ses enfants et critique les autres mères abusant de la gentillesse de la bénévole pendant le séjour vacances: "On fait pas des gamins pour les donner à quelqu'un d'autre pour s'en occuper. »

La séparation du groupe fut souvent une période pénible pour ces parents, arrêt dû généralement à l'âge de l'enfant, arrêt dû plus rarement au départ du thérapeute et à la difficulté du parent à recréer un nouveau lien. Des mères, surtout celles présentant d'importants problèmes de santé mentale, continueront à venir quelques mois au groupe du mardi sans leur enfant âgé de plus de six ans. 


\section{L'aide aux enfants}

Plus de la moitié des parents disent aujourd'hui que les soins reçus ont été utiles pour leur(s) enfant(s). Ceux qui ont suivis des rééducations souvent jusqu'à l'âge de six ans et parfois plus, sont devenus plus ouverts, certains troubles (langage, parole, problèmes de motricité, socialisation) se sont estompés ou ont disparu: " $M r P$. [le kinési] venait à la maison, elle a bien appris à marcher " ; Maintenant elle est moins timide. " ; "Ca fait du bien du contact avec tous ces enfants, ils dessinent, ils s'amusent et les mamans, on parle avec les grandes personnes... J'ai trouvé que la mienne, elle était bien dégourdie... »; «En fait, elle qui parlait pas...ça l'a débloquée quand elle a fait du théâtre avec le CAMSP ; maintenant pour elle, ça se passe très bien, c'est tout remis en ordre "; "Si elle n'a pas posé de problème... c'est sans doute grâce au docteur et aussi au suivi qu'elle a conmu ici. " La recherche a montré par ailleurs que, lorsque les parents venaient régulièrement au groupe, le pourcentage d'absence aux séances individuelles de rééducations des enfants avait diminué, ce qui montre bien que les parents ont compris l'importance de ces soins pour leur enfant. Les effets sur les apprentissages scolaires ont été repérés non seulement par les parents mais aussi par quelques assistantes maternelles que nous avons interviewées. Il en est de même quant à l'amélioration des relations parents-enfants : "L'aînée, quand elle prenait son biberon, elle faisait des rejets et moi, ça m'énervait. Avec la dernière, j'ai jamais eu de problème "; "En fait, ça m'a appris à écouter plus les enfants, qu'avant je ne faisais, et ça m'a permis d'autres relations avec eux par la suite"; "C'était une enfant qui pleurait beaucoup, elle était fort accrochée à sa mère... il fallait toujours la mettre en confiance... Ca l'a aidée au bout d'un an parce que j'ai senti qu'elle s'éloignait de moi. " Dans cette dernière citation, comme dans d'autres notées plus haut, on peut repérer des expressions à connotation psychologique, souvent utilisées par des mères suivies en thérapie (ou encore suivies) depuis longtemps.

Quel que soit le lieu de soin, le discours de certains parents montre leur ambivalence face aux prises en charge. Ainsi, en début d'entretien, une mère mentionne qu'elle ne savait pas pourquoi elle venait avec sa fille et que l'aide n'avait peu duré, puis ultérieurement que ce travail avait duré des années et qu'elle " avait bien accroché avec " la thérapeute. Une violence encore très présente fait jour chez des mères pour lesquelles les soignants sont intervenus pour dénoncer des situations de maltraitance et réintroduire la loi au sein de la famille : "Oui, ici, ça l'a aidé, il est mieux dans sa peau... Mais dans le centre où il a fait du suivi scolaire, ça l'a rendu abruti, c'est pour ça que je l'ai sorti... Y a des choses, j'ai pas digéré, j'ai du mal à digérer " explique une mère à propos d'un lieu de soins qui avait pris le relais de la première institution et dénoncé une situation d'abus sexuel intra-familial. Une autre mère reproche au médecin d'avoir fait un signalement à la suite de ce qui s'était passé lors d'une séance de groupe: "Lui, il avait trop de pouvoir, à cause de cette histoire avec Mme R., elle a dit un mot qu'il fallait pas, les enfants ont dû partir faire des tests et ça a mal tourné ». Cette confrontation avec le signalement ou la perspective d'un placement induit un discours négatif envers le soignant de l'institution.

\section{Pour conclure}

Des familles présentant de graves carences et de nombreux facteurs de vulnérabilité ont été rencontrées plusieurs années après la fin de prises en charge thérapeutiques très précoces et/ou de guidance parentale. Dans ces deux lieux de soins spécifiques, sous-tendus par des références théoriques différentes, les modalités d'interventions et d'accompagnement se sont adaptées au type de situations familiales. Quels que soient les moyens que se sont donnés ces institutions pour réaliser leurs objectifs, on constate qu'aujourd'hui nombre de parents 
assument leur parentalité, la maltraitance physique a disparu, certains sont sortis de l'exclusion sociale. D'une façon générale, on note que c'est approche la plus pragmatique, avec son aspect pédagogique qui a été le mieux perçu par les parents les plus démunis socialement et intellectuellement. D'autres, disposant de plus de ressources intellectuelles, peuvent parler des aspects relationnels du soin psychique.

Dans l'ensemble, le discours de ces parents, surtout des mères, n'est pas très contrasté. Il est positif à l'égard des soins et de l'accompagnement et indique bien les différents domaines dans lesquels les familles disent avoir été aidées. Le fait que ces mères ne se souviennent pas des détails des prises en charge importe peu, ce qui compte c'est qu'elles aient compris le sens de ce qui leur avait été proposé. Ces parents se sont impliqués dans les soins d'autant plus que les relations avec les soignants étaient basées sur la confiance réciproque. Le fait qu'ils se soient sentis traités avec humanité les a aidés dans la restauration d'une certaine estime de soi et de leur image narcissique; se redécouvrant aidables par des soignants qui les ont bien traités, ils ont d'autant plus adhéré aux soins. On mettra aussi à l'actif de ces accompagnements précoces de type thérapeutique ou plus social de longue durée la capacité de ces parents à savoir désormais se faire aider ou demander de 1'aide: "Quand on a une difficulté, à la place de la garder pour soi, on peut aller voir, essayer de les arranger dans les différents organismes "; "Les filles, elles ont plus besoin d'aide, bon ben si j'ai un problème, je peux toujours revenir en parler ».

Annick-Camille DUMARET

Psychologue, ingénieur de recherche à 1'INSERM U. 502 Centre de Recherche Médecine, Sciences, Santé et Société

E-mail : dumaret@vjf.cnrs.fr

\section{Bibliographie}

BLONDEL MP, JARDIN F. «Le suivi indirect dans le cadre d'une unité de soins pour jeunes enfants ». In Interventions psychothérapeutiques parents-jeunes enfants. Guedeney A, Lebovici S (sous la dir.), 1997, Paris, Masson, pp. 15-16.

DAVID M, LAmour M, KREISLER A, HARNish R. "Recherche sur les nourrissons de familles carencées 》. Psychiatrie de l'Enfant, 1984, I: 175-222.

DAVID M, JARDIN F. «Protéger et traiter les bébés exposés à des défaillances parentales. Place de la rencontre interdisciplinaire $\gg$. Devenir, 1993, 5: 19-33.

Donati P., Mollo S., NoRVEZ A., Rollet C. Les centres maternels. Réalités et enjeux éducatifs. L'Harmattan, 1999.

DUMARET A.C., JARDIN F. (collab. PICCHI V., COHEN L., RUFFIN D.) «Soins précoces parents-bébés. Devenir des familles ». Rapport de recherche à la Fondation de Rothschild et à la Fondation de France, nov. $2001,197 p$.

Dumaret A.C., Titran M., DuMONT B. «Soins précoces et prise d'autonomie des familles : tentative d'évaluation ». Colloque International organisé par le Fonds Houtman, Bruxelles, 18-19 avril 2002, Manifeste pour les enfants. Vers un réseau international d'échanges et de bonnes pratiques

DUMARET A.C. Soins médico-sociaux et accompagnement psycho-éducatif. Revue de la littérature étrangère. Archives de Pédiatrie 2003, mai.

DUMONT B. «Aller au delà du regard impossible... Quand ce sont les familles elles mêmes qui font changer le regard du professionnel ». In Accompagner, Erès, 2000.

JARDIN F., DETRY L. «Présentation de 1'Unité de soins spécialisés à domicile de la Fondation de Rothschild du 13è arrondissement ». In Avec Myriam David: quel accueil pour les jeunes enfants? Erès, 2003, 74-84. 
GAUTHIER Y. «Accès à la parentalité de familles vulnérables : peut-on briser la répétition ? » Cahiers de l'Afree, déc. 1998, 19-33.

LAMOUR M, BARRACO M. «Souffrances autour du berceau: Des émotions au soin ». Gaëtan Morin Editeur, Europe, 1998.

TITRAN M. «Témoignage : le groupe du mardi ». Arch. Public Health, 1993, 51, 135-139.

TITRAN M. «Accueillir des parents et enfants en difficultés dans un centre d'action médicosocial précoce », In Santé et apprentissages, La Documentation Française, 1994, 2283-286.

\section{MOTS-CLES}

soins précoces, guidance parentale, familles carencées, exclusion sociale, représentations des soins, évaluation.

\section{NoTES}

1. Ce programme a bénéficié de l'infrastructure du CERMES. Il a été subventionné par la Fondation de France, la Fondation de Rothschild (convention n ${ }^{\circ} 257-8 \mathrm{DH} 17 \mathrm{~F}$ ), 1'Oeuvre Grancher, la Fondation Mac Donald, les Conseils Généraux du Nord et du Pas-de-Calais, la Région Nord Pas-de-Calais.

2. Le discours de familles venues à la suite de problèmes périnataux graves, de familles sans symptomatologie définie présentant principalement des troubles de la parentalité et celui des mères psychotiques chroniques, n'a pas été repris dans ces analyses. Ceci a permis de rendre plus homogène le milieu socio-culturel dans lequel a été produit ce discours.

3. Il peut arriver qu'une mère soit exclue du groupe du mardi à cause de ses comportements de mise en danger de son enfant ou d'agression envers les autres parents.

4. Le manque de respect des confidences échangées, leur divulgation hors du lieu où elles ont été produites sont source de rupture dans la confiance entre l'usager et l'équipe professionnelle. La question des limites du «secret professionnel » est discutée en permanence dans les institutions.

5. Il s'agit de parents qui perçoivent l'allocation adulte handicapé (AAH) et dont la situation sociofamiliale et les problèmes de santé mentale se sont stabilisés. 